\title{
Sensibilidade ao Contraste em Crianças Pré-Escolares com Método Psicofísico $^{1}$
}

\author{
Valtenice de Cássia Rodrigues de Matos França \\ Natanael Antonio dos Santos ${ }^{2}$ \\ Liana Chaves Mendes \\ Universidade Federal da Paraíba
}

\begin{abstract}
RESUMO - O objetivo deste trabalho foi utilizar o método psicofísico da escolha forçada para mensurar a função de sensibilidade ao contraste para freqüências espaciais (FSC) na faixa de 0,25 a $2 \mathrm{cpg}$, em crianças pré-escolares. Foram estimados limiares de contraste para 15 participantes (10 crianças e cinco adultos). Os resultados mostraram que as curvas de sensibilidade (FSC) de crianças de 4 e 5 anos e adultos apresentam perfis gerais semelhantes, embora sejam diferentes entre si. Estes resultados sugerem que o método psicofísico da escolha forçada pode ser utilizado para mensurar a FSC de crianças a partir dos 4 anos.
\end{abstract}

Palavras-chave: sensibilidade ao contraste; desenvolvimento visual; criança pré-escolar; freqüência espacial; método da escolha forçada.

\section{Contrast Sensitivity in Preschool Children with Psychophysical Method}

\begin{abstract}
The aim of this work was to use the psychophysical forced-choice staircase method to measure contrast sensitivity function for spatial frequencies (CSF) in the range between 0.25 and $4 \mathrm{cpd}$ in preschool children and adults. We estimated the contrast thresholds of 15 participants ( 10 children and five adults). The results showed that the sensitivity curves (CSF) of 4 and 5 years old chidren and adults were similar in general profiles although they were different between themselves. These results suggest that the psychophysical forced-choice staircase method can be used to measure the CSF of children over 4 years old.
\end{abstract}

Key words: contrast sensitivity; visual development; preschool child; spatial frequency; forced-choice staircase method.

A mensuração da função de sensibilidade ao contraste (FSC) é considerada uma das técnicas mais completas e poderosas na avaliação teórica e clínica das funções visuais (Adams \& Courage, 2002; Richman \& Lyons, 1994; Van Sluyters, Atkinson, Held, Hoffman \& Shatz, 1990). A sensibilidade ao contraste é definida como a recíproca do limiar de contraste (1/FSC) que corresponde à quantidade mínima de contraste necessária para detectar um objeto qualquer de uma determinada freqüência espacial. $O$ contraste é a relação entre a diferença da luminância máxima e luminância mínima dividido pela soma das duas.

No presente estudo, utilizamos o método psicofísico da escolha forçada e estímulos visuais do tipo grade senoidal vertical (ou freqüência espacial) para mensurar a FSC de crianças de 4 e 5 anos. Mensuramos também a FSC de adultos jovens para efeito de comparação.

O objetivo foi utilizar o método psicofísico da escolha

1 Trabalho apresentado na XX Reunião Anual da Federação de Sociedades de Biologia Experimental (FeSBE). Pesquisa financiada pelo CNPq através de Auxílio Individual (processo: 477258/2001-8), Bolsa IC/ PIBIC/UFPB/CNPq e Bolsa de Produtividade em Pesquisa (processo: 307182/2003-7).

2 Endereço: Universidade Federal da Paraíba, Depto. de Psicologia, CCHLA - UFPB, Campus I, Cidade Universitária, João Pessoa, PB, Brasil, 58051-900.E-mail: natanael_labv@yahoo.com.br forçada para avaliar a FSC de crianças pré-escolares. Estudos desta natureza com crianças são raros na literatura e inéditos no Brasil (Benedek, Benedek, Kéri \& Janáky, 2003). Participaram dos experimentos 15 voluntários distribuídos igualmente em três faixas etárias (4 anos, 5 anos e 19-23 anos). Todos os participantes apresentavam acuidade visual normal ou corrigida.

Os estímulos foram gerados num monitor de vídeo monocromático CLINTON MEDICAL de 21 polegadas, tela plana, digital, de alta resolução, controlado por um microcomputador. As medições foram feitas à distância de $150 \mathrm{~cm}$ da tela do monitor com visão binocular. A luminância média da tela foi de $0,7 \mathrm{~cd} / \mathrm{m}^{2}$ ajustada por um fotômetro do tipo SPOT METTER, com precisão de um grau, ASAHI PENTAX. A luminância mínima da tela foi de $0,5 \mathrm{~cd} / \mathrm{m}^{2}$ e a máxima de $0,9 \mathrm{~cd} / \mathrm{m}^{2}$.

Os estímulos de freqüência espacial acromática utilizados para mensurar a FSC foram 0,25; 0,5; 1 e $2 \mathrm{cpg}$. Estes eram circulares e tinham um diâmetro de 7 graus de ângulo visual (Figura 1). Freqüência espacial corresponde ao número de ciclos (ou listras claras e escuras) por grau de ângulo visual (cpg).

As estimativas foram realizadas com o método psicofísico da escolha forçada (Santos, Simas \& Nogueira, 2003, 2004; Santos, Nogueira \& Simas, 2005). Este método se baseia no 


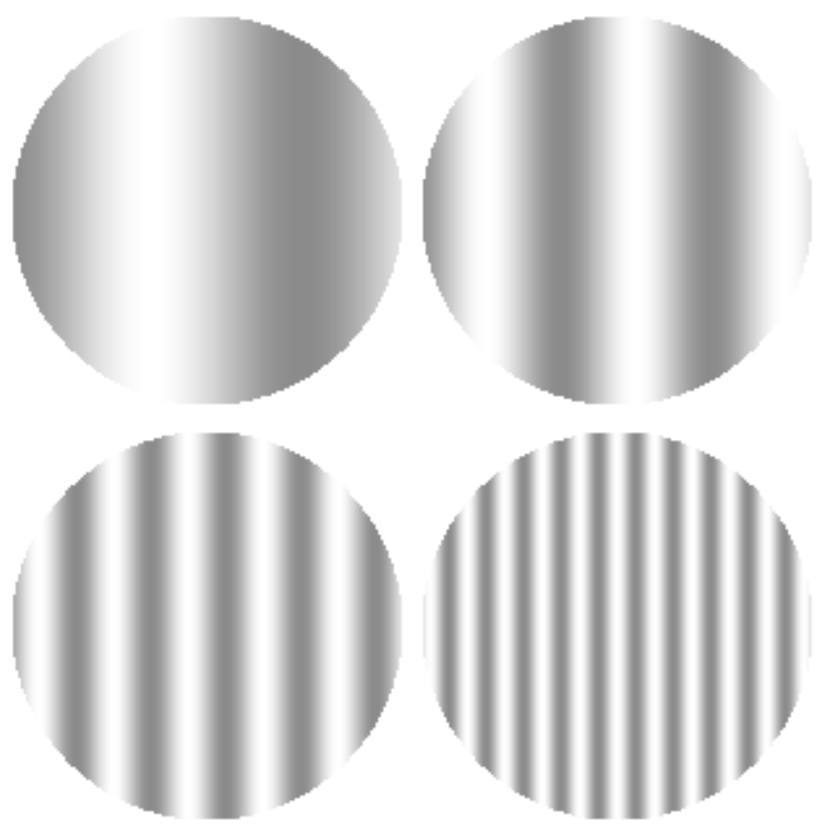

Figura 1. Exemplos de estímulos de frequiências espaciais (acima, da esquerda para a direita, 0,25 e $0,5 \mathrm{cpg}$ e embaixo, da esquerda para direita, 1 e $2 \mathrm{cpg}$ ).

Estímulos originalmente calibrados para serem vistos a $150 \mathrm{~cm}$ de distância.

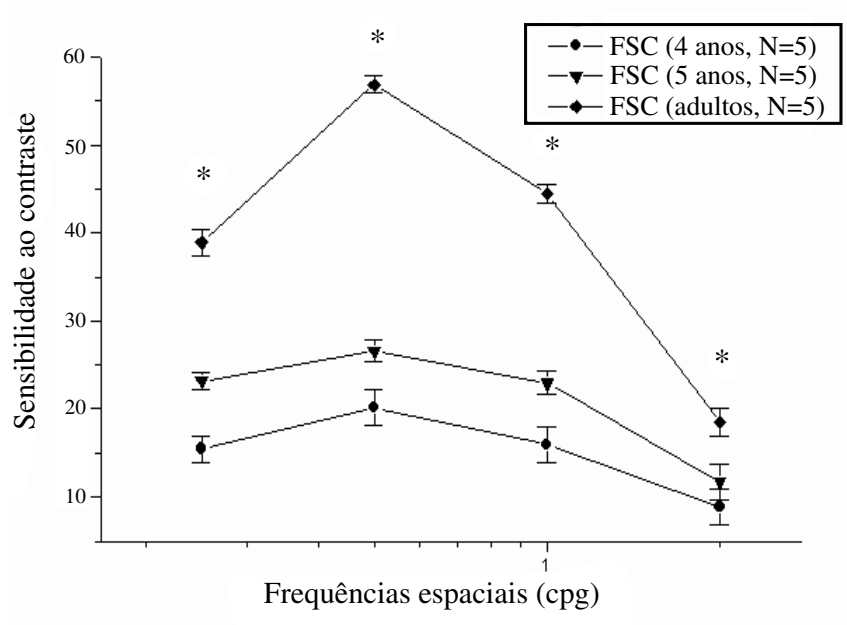

Figura 2. Funções de sensibilidade ao contraste de crianças de 4 e 5 anos e adultos para freqüências espaciais. As linhas verticais mostram o erro padrão da média para cada frequiência $(0,25 ; 0,5 ; 1$ e $2 \mathrm{cpg})$.

$* P<0,001$ mostrou diferença significativa entre todas as idades em todas as frequiências (teste post-hoc Tukey HSD).

cálculo da probabilidade de acertos consecutivos por parte do voluntário, ou seja, em cerca de 100 apresentações de escolhas entre os dois estímulos (estímulo de teste e estímulo neutro), o estímulo de teste (ou freqüência espacial) é percebido $79 \%$ das vezes pelo voluntário. O estímulo neutro foi sempre um padrão homogêneo com luminância média de $0,7 \mathrm{~cd} / \mathrm{m}^{2}$. O procedimento para medir o limiar para cada frequiência consistiu na apresentação sucessiva simples do par de estímulos e o voluntário teria que escolher dentre eles qual continha a freqüência espacial. O critério adotado para variar o contraste da frequiência de teste era o de três acertos consecutivos para decrescer uma unidade e um erro para acrescer da mesma unidade $(0,08 \%)$. A sessão experimental terminou automaticamente ao completar cinco reversões.

A sessão experimental iniciou com um sinal sonoro seguido imediatamente pela apresentação do primeiro estímulo por $2 \mathrm{~s}$, seguido de um intervalo entre estímulos de $1 \mathrm{~s}$, seguido pela apresentação do segundo estímulo por $2 \mathrm{~s}$ e da resposta do voluntário. A ordem de apresentação dos estímulos era aleatória. Se a resposta do voluntário fosse correta, era seguida por outro sinal sonoro e um intervalo de $3 \mathrm{~s}$ para a sequiência se repetir. A resposta foi pressionar o botão do lado esquerdo do mouse quando o estímulo de teste fosse apresentado primeiro e, o botão do lado direito quando fosse apresentado em segundo lugar, isto é, após o estímulo neutro.

Cada uma das frequiências da FSC foi estimada pelo menos duas vezes (duas sessões experimentais), em dias diferentes, por cada um dos voluntários. No total, 10 curvas foram medidas para cada grupo de voluntários.

A Figura 2 mostra a FSC de crianças de 4 e 5 anos e adultos. A sensibilidade ao contraste é mostrada em função da frequiência espacial. As barras verticais indicam os erros padrões das médias. A FSC é a recíproca da curva de limiar de contraste (1/FSC). Logo, quanto menor o limiar de contraste maior a sensibilidade do sistema visual humano e vice-versa. Assim, os menores valores de limiares correspondem aos maiores valores de sensibilidade ao contraste.

Os resultados mostram que a sensibilidade máxima ocorreu na freqüência espacial de $0,5 \mathrm{cpg}$ para todas as idades (Figura 2). Entretanto, a sensibilidade das crianças de 4 anos na frequiência de $0,5 \mathrm{cpg}$ foi da ordem de 1,3 vezes menor do que a das crianças de 5 anos, que foi da ordem de 2,1 vezes menor do que as dos adultos. A ANOVA para medidas repetidas revelou diferenças significativas entre as idades $\left[F_{(2,297)}=157,2 ; p<0,001\right]$, entre as freqüências $\left[F_{(3,891)}=415,1 ; \mathrm{p}<0,001\right]$ e uma interação entre freqüências e idades $\left[F_{(6,891)}=34,1 ; p<0,001\right]$. As análises com o teste post-hoc Tukey HSD mostraram diferenças significativas entre a FSC de crianças de 4 e 5 anos $(p<0,001)$, crianças de 5 anos e adultos $(p<0,001)$ e crianças de 4 anos e adultos $(p<0,001)$. No geral, estes resultados mostram que a FSC melhora gradativamente e de forma significativa com o aumento da idade.

Os nossos resultados mostraram ainda que o método psicofísico da escolha forçada, geralmente utilizado nas pesquisas com adultos, pode ser utilizado de forma confiável para mensurar a FSC de crianças a partir dos 4 anos (Figura 2). A curva de sensibilidade das crianças de 4 anos é consistente uma vez que ela apresenta um perfil geral muito semelhante às curvas de sensibilidade (FSC) de crianças de 5 anos e adultos. A diferença entre a FSC das crianças de 4 anos, 5 anos e adultos pode ser relacionada ao domínio da sensibilidade ao contraste que aumenta gradativamente com o aumento da idade. Por outro lado, nós não conseguimos mensurar a FSC para crianças com menos de 4 anos (três 
crianças com idades entre 40-47 meses não conseguiram realizar os experimentos com sucesso). É possível relacionar a falta de sucesso das crianças com menos de 4 anos ao tipo de tarefa e as características do método psicofísico utilizado (Benedek e cols., 2003; Bradley \& Freeman, 1982; Ellemberg, Lewis, Liu \& Maurer, 1999). Pois, Richman e Lyons (1994) conseguiram mensurar a FSC de crianças de 3 anos com método psicofísico e procedimento diferente. Estes autores utilizaram um procedimento no qual os estímulos eram apresentados em cartões (Vistech Chart) e a criança precisava apenas olhar ou apontar na direção do cartão que continha a grade. Este aspecto pode explicar porque esses autores conseguiram mensurar a FSC para crianças mais jovens.

Embora a mensuração da FSC de crianças seja mais comum com potencial visual evocado (Norcia \& Tyler, 1985; Norcia, Tyler \& Hamer, 1990). Baseado em nossos resultados e em dois outros estudos (Richman \& Lyons, 1994; Bradley \& Freeman, 1982), pode-se concluir que é possível utilizar o método psicofísico da escolha forçada para caracterizar e acompanhar a resposta do sistema visual de crianças pré-escolares a partir dos 4 anos. É com essa perspectiva que prosseguiremos com as nossas investigações.

\section{Referências}

Adams, R. J. \& Courage, M. L. (2002). Using a single test to measure human contrast sensitivity from early childhood to maturity. Vision Research, 42, 1205-1210.

Benedek, G., Benedek, K., Kéri, S. \& Janáky, M. (2003). The scotopic low-frequency spatial contrast sensitivity develops in children between the ages of 5 and 14 years. Neuroscience Letters, 345, 161-164.

Bradley, A. \& Freeman, R. D. (1982). Contrast sensitivity in children. Vision Research, 22, 953-959.

Ellemberg, D., Lewis, T. L., Liu, C. H. \& Maurer, D. (1999). Development of spatial and temporal vision during childhood. Vision Research, 39, 2325-2333.
Norcia, A. M. \& Tyler, C. W. (1985). Spatial frequency sweep VEP: Visual acuity during the first year of life. Vision Research, 25, 1399-1408.

Norcia, A. M., Tyler, C. W. \& Hamer, R. D. (1990). Development of contrast sensitivity in the human infant. Vision Research, 30(10), 1475-1486.

Richman, J. E. \& Lyons, S. (1994). A forced choice procedure for evaluation of contrast sensitivity function in preschool children. Journal of the American Optometric Association, 65(12), 859-864.

Santos, N. A., Simas, M. L. B. \& Nogueira, R. M. T. B. L. (2003). Processamento visual da forma em idosos: Curvas de limiar de contraste para frequiências angulares e senoidais. Psicologia: Reflexão \& Crítica, 16(2), 271-277.

Santos, N. A., Simas, M. L. B. \& Nogueira, R. M. T. B. L. (2004). Comparison of angular frequency contrast sensitivity in Young and older adults. Brazilian Journal of Medical and Biological Research, 37, 375-378.

Santos, N. A., Nogueira, R. M. T. L. \& Simas, M. L. B. (2005). Processamento visual da forma: Evidências para canais múltiplos de frequiências angulares em humanos. Psicologia Reflexão e Crítica, 18(1), 98-103.

Van Sluyters, R. C., Atkinson, M. S., Held, R. M., Hoffman, K. \& Shatz, C. J. (1990). The development of vision and visual perception. Em S. W. Spillmann \& J. S. Werner (Orgs.), The Neurophysiological Foundations (pp. 349-379). New York: Academic Press. 


\title{
VIII COGRESSO NACIONAL DE PSICOLOGIA ESCOLAR E EDUCACIONAL
}

\author{
História, Compromissos e Perspectivas
}

Data: 26 a 29 de Abril de 2007

Local: São João Del Rei - MG

Organização

Associação Brasileira de Psicologia Escolar e Educacional (ABRAPEE)

Universidade Federal de São João Del-Rei - UFSJ

Informações e inscrições

http://www.abrapee.psc.br

e-mail: abrapee@abrapee.psc.br 